\title{
Existence of solutions to a class of generalized vector variational-like inequalities in fuzzy environment
}

\author{
Wang Chao ${ }^{1, a}$, Zhao Ya-li, ${ }^{1,{ }^{*}}$ and Han Dong-xue ${ }^{1, c}$ \\ ${ }^{1}$ College of Physics and Mathematics, Bohai University, \\ Jinzhou, Liaoning 121013, P.R.China \\ abdywangchao@163.com, byalizhao2000@163.com, dongxuehan2014@163.com
}

Keywords: Generalizd vector variational-like inequality. Fuzzy environment. KKM theorem. Upper semicontinuous. Lower semicontinuous.

Abstract. In this paper, we introduce and study a class of generalized vector variational-like inequality problems in fuzzy environment. Under suitable conditions, we get the existence of the solution to the generalized vector variational like inequalities in fuzzy environment by using the known Fan-KKM theorem.

\section{Introduction}

Since the 1960s, there have been many literatures on the variational inequality problems attracting more and more attention. The concept of fuzzy set has penetrated almost all branches of mathematics ever since the introduction of fuzzy sets by L.A.Zaden [11]. In 1989, Zhang and Zhu[2] introduced the concept of variational inequality for fuzzy mapping in abstract space and investigated the existence problem of solutions to some class of variational inequalities for fuzzy mapping. Zhang et al. applied maximal element lemma[1] to prove the existence of the solutions to the generalized vector variational inequality problem in 2014. Ahmad et al. [7] obtained the existence theorem for the fuzzy mixed vector F-variational inequalities. Ding et al. [8] studied about fuzzy generalized vector variational inequalities and complementarity problem. Khan et al. [9] studied about a fuzzy extension of generalized multivalued $\eta$-mixed vector variational-like inequalities on locally convex Hausdoff topological vector spaces.

Inspired and motivated by the above research work, by making use of the Fan - KKM theorem, we prove the existence theorems of the solution to the generalized vector variational-like inequalities in fuzzy environment. The results obtained here extend the corresponding results of [1].

\section{Preliminaries}

In this paper, let $Z$ be a locally convex Hausdorff topological vector space, $E$ be a Hausdorff topological vector space. $L(E, Z)$ denotes the space of all continuous linear operators from $E$ to $Z,\langle l, x\rangle$ denotes the evaluation of $l \in L(E, Z)$ at $x \in E$. If $L(E, Z)$ is equipped with $\sigma$-topology, we know that $L(E, Z)$ becomes a locally convex space from the Corollary in the literature [3]. It follows from Ding and Tarafdar [4], the bilinear mapping $\langle\cdot \cdot\rangle: L(E, Z) \times E \rightarrow Z$ is continuous. Let $K$ be a nonempty convex subset of a Hausdorff topological vector space $E, C: K \rightarrow 2^{Z}$ be a set-valued mapping such that for any $x \in K, C(x) \subset Z$ is a closed pointed convex cone and int $C(x) \neq \phi(\forall x \in K)$. Partial order relation in $Z$ is defined with the convex cone $C(x)$ as follows :

$y_{1} \leq_{C(x)} y_{2} \Leftrightarrow y_{2}-y_{1} \in C(x), \forall y_{1}, y_{2} \in Z$. Let $\theta: K \times K \rightarrow E, g: K \rightarrow K, \eta: K \times K \rightarrow Z$, $N: L(E, Z) \times L(E, Z) \times L(E, Z) \rightarrow L(E, Z)$ be four single-valued mappings. Let $M, S, T: K \rightarrow f(L(E, Z))$ be three fuzzy mappings on $2^{L(E, Z)}$, and let $a, b, c: K \rightarrow[0,1]$ be three real functions. 
Definition 2.1(i)The generalized vector variational-like inequality problem in fuzzy environment(for short, denoted by GVVLIFE) is to find $x \in K, u \in\left(M_{x}\right)_{a(x)}, v \in\left(S_{x}\right)_{b(x)}, w \in\left(T_{x}\right)_{c(x)}$ such that

$$
\langle N(u, v, w), \theta(y, g(x))\rangle+\eta(g(x), y) \notin-\operatorname{int} C(x), \forall y \in K .
$$

(ii)If $\theta(y, g(x))=y-g(x), \forall(x, y) \in K \times K$, then the GVVLIFE is reduced to the generalized vector variational inequality problem in fuzzy environment(for short, denoted by GVVIFE): Find $x \in K, u \in\left(M_{x}\right)_{a(x)}, v \in\left(S_{x}\right)_{b(x)}, w \in\left(T_{x}\right)_{c(x)}$ such that

$$
\langle N(u, v, w), y-g(x)\rangle+\eta(g(x), y) \notin-\operatorname{int} C(x), \forall y \in K .
$$

Definition $2.2^{[5]}$ int $A$ denotes interior of $A$ and $c o A$ denotes convex hull of $A$. Let $X$ and $Y$ be two Hausdorff topological vector spaces, $G: X \rightarrow 2^{Y}$ be set-valued mapping, $G^{-1}(y)=\{x \in X \mid y \in G(x)\}$.

(i)If $G(X)$ is compact for any $x \in X$, then $G$ is upper semicontinuous at $x_{0}$ if and only if for any net $\left\{x_{\alpha}\right\} \subset X$ such that $x_{\alpha} \rightarrow x$ and if any $y_{\alpha} \in G\left(x_{\alpha}\right)$, there exists $y_{0} \in G\left(x_{0}\right)$ and a subset $\left\{y_{\beta}\right\} \subset\left\{y_{\alpha}\right\}$ such that $y_{\beta} \rightarrow y_{0}$.

(ii) $G$ is lower semicontinuous at $x_{0}$ if and only if for any net $\left\{x_{\alpha}\right\} \subset X$ with $x_{\alpha} \rightarrow x$, and for any $y \in G(x)$, there exist a net $\left\{y_{\alpha}\right\}$ such that $y_{\alpha} \in G\left(x_{\alpha}\right)$ and $y_{\alpha} \rightarrow y$.

(iii) $G$ is closed, if for any net $\left\{x_{\alpha}\right\} \subset X$ and $x_{\alpha} \rightarrow x$, any net $\left\{y_{\alpha}\right\} \subset Y$, such that $y_{\alpha} \rightarrow y$ and $y_{\alpha} \in G\left(x_{\alpha}\right)$ for any $\alpha$, then we have $y \in G(x)$, it says that $G$ have closed set. If the graph of $G, G r(G)=\{(x, y) \in X \times Y: y \in G(x)\}$ is closed in $X \times Y$.

Definition 2.3 Let $X$ and $Y$ be Hausdorff topological vector space, and $T: X \rightarrow f(Y)$ be a fuzzy mapping, $T$ is said to have fuzzy set-valued, if $T_{x}(Y)$ is upper semicontinuous on $X \times Y$ as a real ordinary function.

Lemma 2.4 Let $K$ be a nonempty closed convex subset of a real Hausdorff topological vector space $X, E$ be a nonempty closed convex subset of a real Hausdorff topological vector space $Y$. Let $a: X \rightarrow[0,1]$ be a lower semicontinuous function. Let $T: K \rightarrow f(E)$ be a fuzzy mapping such that $\left(T_{x}\right)_{a(x)} \neq \phi$ for any $x \in X, \widetilde{T}: K \rightarrow 2^{E}$ be a multifunction defined by $\widetilde{T}=\left(T_{x}\right)_{a(x)}$. If $T$ is a closed set- valued mapping, then $\tilde{T}$ is a closed multifunction .

Definition 2.5 Let $f_{1}: K \times K \rightarrow Z$ be a mapping. It is said to be convex in the second argument if and only if for any $x \in K$, we have

$$
f_{1}\left(x, \sum_{i=1}^{n} t_{i} y_{i}\right) \in \sum_{i=1}^{n} t f_{1}\left(x, y_{i}\right)-C(x), \forall y_{i} \in K, t_{i} \in[0,1](i=1, \mathrm{~L}, n), \sum_{i=1}^{n} t_{i}=1 .
$$

Similarly, we can give the definition of convexity of the mapping in the first argument.

Difinition 2.6 Let $f_{1}: K \times K \rightarrow Z$ be a mapping. It is said to be affine in the first argument if and only if for any $x \in K$, we have

$$
f_{2}\left(\sum_{i=1}^{n} t_{i} y_{i}, x\right)=\sum_{i=1}^{n} t f_{2}\left(y_{i}, x\right), \forall y_{i} \in K, t_{i} \in R(i=1, \mathrm{~L}, n), \sum_{i=1}^{n} t_{i}=1 .
$$

Similarly, we can give the affine definition of in the second argument.

Definition 2.7(KKM mapping) Let $X$ be a Hausdorff topological vector space. $E \subset X$ be a nonempty subset. Let $F: E \rightarrow 2^{X}$ be set-valued mapping, $F$ is said to be a KKM mapping, if $c o\left\{x_{1}, \mathrm{~L} x_{n}\right\} \subset \bigcup_{i=1}^{n} F(x)$ for every finite subset $\left\{x_{1}, \mathrm{~L} x_{n}\right\}$ in $X$.

Lemma $\mathbf{2 . 8}^{[\mathbf{6}]}$ (Fan-KKM theorem) Let $X$ be a Hausdorff topological vector space, $E$ be a nonempty subset in $X$. Let $F: E \rightarrow 2^{X}$ be a KKM mapping and $F(x)$ be closed for every $x \in X$, 
and there exists a $x^{*} \in E$ such that $F\left(x^{*}\right)$ be compact, then $\left.\right|_{x \in E} F(x) \neq \phi$.

Definition 2.9 Let $K$ be a nonempty compact convex subset, $(N, \theta, \eta)$ is said to be locally non-positive respect to $(M, S, T, g)$ at $x_{0} \in K$, if there exists a neighbourhood $V\left(x_{0}\right)$ of $x_{0}$ and a point $y_{0} \in K I$ int $V\left(x_{0}\right)$, such that $u \in\left(M_{x}\right)_{a(x)}, v \in\left(S_{x}\right)_{b(x)}, w \in\left(T_{x}\right)_{c(x)}$ and

$$
\left\langle N(u, v, w), \theta\left(y_{0}, g(x)\right)\right\rangle+\eta\left(g(x), y_{0}\right) \in-C(x), \forall x \in K \cap b d V\left(x_{0}\right) .
$$

If $g: E \rightarrow E$ is the identity mapping, we say that $(N, \theta, \eta)$ is locally non-positive at $x_{0} \in K$ respect to $(M, S, T)$, if there exists a neighbourhood $V\left(x_{0}\right)$ of $x_{0}$ and a point $y_{0} \in K \mathrm{I}$ int $V\left(x_{0}\right)$, such that $u \in\left(M_{x}\right)_{a(x)}, v \in\left(S_{x}\right)_{b(x)}, w \in\left(T_{x}\right)_{c(x)}$ and

$$
\left\langle N(u, v, w), \theta\left(y_{0}, x\right)\right\rangle+\eta\left(x, y_{0}\right) \in-C(x), \forall x \in K \cap b d V\left(x_{0}\right) .
$$

\section{Existence of the GVVLIFE}

Theorem 3.1 Let $Z$ be a locally convex Hausdorff space, $K$ be a nonempty convex subset of a Hausdorff topological vector space $E, L(E, Z)$ be a space equipped with $\sigma$-topology. Let $\tilde{M}, \widetilde{S}, \widetilde{T}: K \rightarrow 2^{L(E, Z)}$ be three upper semicontinuous compact-valued mappings that be induced by fuzzy mapping $M, S, T: K \rightarrow f(L(E, Z)$, respectively, that is,

$\tilde{M}(x)=\left(M_{x}\right)_{a(x)}, \tilde{S}(x)=\left(S_{x}\right)_{b(x)}, \widetilde{T}(x)=\left(T_{x}\right)_{c(x)}$, for any $x \in K$. Let $\eta: K \times K \rightarrow Z, \theta: K \times K \rightarrow E$, $g: K \rightarrow K, N: L(E, Z) \times L(E, Z) \times L(E, Z) \rightarrow L(E, Z)$ be four single-valued mappings.

If the following conditions are satisfied:

(i) $\eta$ is convex in the second argument and continuous in the first argument, and $\eta(g(x), x)=0$ for any $x \in K$;

(ii) $\theta$ is affine in the first argument and continuous in the second argument, and $\theta(x, g(x))=0$ for any $x \in K$;

(iii) $g$ is continuous and affine;

(iv) $C: K \rightarrow 2^{Z}$ is a multifunction such that for any $x \in K, C(x) \subset Z$ is a closed pointed convex cone and int $C(x) \neq \varnothing$, and $G: K \rightarrow 2^{Z}$ is a multifunction defined by $G(x)=Z \backslash(-\operatorname{int} C(x))$ for any $x \in K$;

(v) there exsits a nonempty compact subset $A$ of $K$ and a nonempty compact convex subset $B$ of $K$ such that for any $x \in K \backslash A, \bar{y} \in B$, and

$$
\langle N(u, v, w), \theta(\bar{y}, g(x))\rangle+\eta(g(x), \bar{y}) \in-\operatorname{int} C(x),
$$

where $u \in\left(M_{x}\right)_{a(x)}, v \in\left(S_{x}\right)_{b(x)}, w \in\left(T_{x}\right)_{c(x)}$. Then the GVVLIFE has a solution.

Proof. Let $P: K \rightarrow 2^{K}$ be a set valued mapping and defined by as follows:

$$
P(y)=\{x \in K \mid\langle N(u, v, w), \theta(y, g(x))\rangle+\eta(g(x), y) \notin-\text { int } C(x)\}, \forall y \in K .
$$

It is obvious that any $x^{*} \in \mathrm{I}_{y \in K} P(y)$ is a solution to the GVVLIFE, so we only need to prove that $\prod_{y \in K} P(y) \neq \phi \cdot$

First, we prove that $P(y)$ is closed for any $y \in K$. Let the net $\left\{x_{\alpha}\right\} \subset P(y)$ be such that $x_{\alpha} \rightarrow x$. Since $K$ is closed, then $x \in K$. It follows from $x_{\alpha} \in P(y)$ for any $\alpha$ that

which implies

$$
\left\langle N(u, v, w), \theta\left(y, g\left(x_{\alpha}\right)\right)\right\rangle+\eta\left(g\left(x_{\alpha}\right), y\right) \notin-\operatorname{int} C\left(x_{\alpha}\right),
$$

From Conditions (ii) and (iii) that 


$$
\left\langle N(u, v, w), \theta\left(y, g\left(x_{\alpha}\right)\right)\right\rangle+\eta\left(g\left(x_{\alpha}\right), y\right) \rightarrow\langle N(u, v, w), \theta(y, g(x))\rangle+\eta(g(x), y) .
$$

In view of Condition (iv), we have

$$
\langle N(u, v, w), \theta(y, g(x))\rangle+\eta(g(x), y) \notin-\operatorname{int} C(x) .
$$

Then $P(y)$ is closed. Again by Condition (v), we have $P(y) \subseteq A$. Thus $P(y)$ is compact subset of A.

Next, we prove that $P$ is a KKM mapping. Suppose that there exists a finite subset $\left\{y_{1}, y_{2}, \mathrm{~L}, y_{n}\right\}$ of $K, t_{i} \geq 0(i=1,2, \mathrm{~L}, n), \sum_{i=1}^{n} t_{i}=1$, such that $y^{*}=\sum_{i=1}^{n} t_{i} y_{i} \notin \bigcup_{i=1}^{n} P\left(y_{i}\right)$, which means that

$$
\left\langle N(u, v, w), \theta\left(y_{i}, g\left(y^{*}\right)\right)\right\rangle+\eta\left(g\left(y^{*}\right), y_{i}\right) \in-\operatorname{int} C\left(y^{*}\right) .
$$

It follows from Conditions (i) and (ii), we have

$$
\begin{aligned}
& \left\langle N(u, v, w), \theta\left(\sum_{i=1}^{n} t_{i} y_{i}, g\left(y^{*}\right)\right)\right\rangle+\eta\left(g\left(y^{*}\right), \sum_{i=1}^{n} t_{i} y_{i}\right) \\
& \in \sum_{i=1}^{n} t_{i}\left\langle N(u, v, w), \theta\left(y_{i}, g\left(y^{*}\right)\right)\right\rangle+\sum_{i=1}^{n} t_{i} \eta\left(g\left(y^{*}\right), y_{i}\right)-C\left(y^{*}\right),
\end{aligned}
$$

which implies that

$$
\left\langle N(u, v, w), \theta\left(y_{i}, g\left(y^{*}\right)\right)\right\rangle+\sum_{i=1}^{n} t_{i} \eta\left(g\left(y^{*}\right), y_{i}\right)-C\left(y^{*}\right) \in-\operatorname{int} C\left(y^{*}\right) .
$$

Noting $\theta\left(y^{*}, g\left(y^{*}\right)\right)=0$, so we get $\sum_{i=1}^{n} t_{i} \eta\left(g\left(y^{*}\right), y_{i}\right)-C\left(y^{*}\right) \in-\operatorname{int} C\left(y^{*}\right)$, which contradicts with $\eta\left(g\left(y^{*}\right), y^{*}\right)=0$. So $P$ is a KKM mapping. By Lemma 2.8, $\underset{x \in K}{ } P(x) \neq \phi$, the GVVLIFE has a solution, which completes the proof.

Corollary 3.2 Let $Z$ be a locally convex Hausdorff space, $K$ be a nonempty convex subset of a Hausdorff topological vector space $E, L(E, Z)$ be a space equipped with $\sigma$-topology. Let $\widetilde{M}, \widetilde{S}, \widetilde{T}: K \rightarrow 2^{L(E, Z)}$ be three upper semicontinuous compact valued mappings that be induced by fuzzy mapping $M, S, T: K \rightarrow f\left(L(E, Z)\right.$, respectively that $\tilde{M}(x)=\left(M_{x}\right)_{a(x)}, \tilde{S}(x)=\left(S_{x}\right)_{b(x)}$, $\tilde{T}(x)=\left(T_{x}\right)_{c(x)}$, for any $x \in K$.Let $\eta: K \times K \rightarrow Z, \theta: K \times K \rightarrow E, g: K \rightarrow K$, $N: L(E, Z) \times L(E, Z) \times L(E, Z) \rightarrow L(E, Z)$ be four single-valued mappings.

If the following conditions are satisfied:

(i) $\eta$ is convex in the second argument and continuous in the first argument, and $\eta(g(x), x)=0$ for any $x \in K$;

(ii) $g$ is continuous and affine, $x-g(x)=0$ for any $x \in K$;

(iii) $C: K \rightarrow 2^{Z}$ is convex and $G: K \rightarrow 2^{Z}$ is defined that $G(x)=Z \backslash(-$ int $C(x))$ is closed for any $x \in K$;

(iv)there exsits a nonempty compact subset $A$ of $K$ and a nonempty compact convex subset $B$ of $K$ such that for any $x \in K \backslash A, \bar{y} \in B$ and

$$
\langle N(u, v, w), \bar{y}-g(x)\rangle+\eta(g(x), \bar{y}) \in-\operatorname{int} C(x),
$$

where $u \in\left(M_{x}\right)_{a(x)}, v \in\left(S_{x}\right)_{b(x)}, w \in\left(T_{x}\right)_{c(x)}$. Then the GVVIFE has a solution.

Proof. Similar to the proof of Theorem 3.1, so it is omitted, then completing the proof.

Theorem 3.3 Let $Z$ be a locally convex Hausdorff space, $K$ be a nonempty convex subset of a Hausdorff topological vector space $E, L(E, Z)$ be a space equipped with $\sigma$-topology. Let $\widetilde{M}, \widetilde{S}, \widetilde{T}: K \rightarrow 2^{L(E, Z)}$ be three upper semicontinuous compact valued mappings that be induced by fuzzy mapping

$M, S, T: K \rightarrow f(L(E, Z)$ respectively that 
$\tilde{M}(x)=\left(M_{x}\right)_{a(x)}, \tilde{S}(x)=\left(S_{x}\right)_{b(x)}, \quad \tilde{T}(x)=\left(T_{x}\right)_{c(x)}, \quad$ for $\quad$ any $\quad x \in K \quad$.Let $\quad \eta: K \times K \rightarrow Z$, $\theta: K \times K \rightarrow E, g: K \rightarrow K, \quad N: L(E, Z) \times L(E, Z) \times L(E, Z) \rightarrow L(E, Z)$ be four single-valued mappings. If the following conditions are satisfied:

(i) $\theta(x, g(x))=0, \eta(g(x), x)=0, \forall x \in K$;

(ii) $\theta, \eta$ and $g$ are continuous;

(iii) $\theta$ is affine in the first argument, $\eta$ is convex in the second argument;

(iv) $G: K \rightarrow 2^{Z}$ is defined that $G(x)=Z \backslash(-$ int $C(x))$ is closed for any $x \in K$;

(v) $(N, \theta, \eta)$ is said to be locally non-positive respect to $(M, S, T, g)$ at $x_{0} \in K$ and there exists a nonempty compact convex subset $B \subseteq K \mid V\left(x_{0}\right), y_{0} \in B$, such that

$$
\left\langle N(u, v, w), \theta\left(y_{0}, g(x)\right)\right\rangle+\eta\left(g(x), y_{0}\right) \in-\operatorname{int} C(x), \forall x \in\left(K \mid V\left(x_{0}\right)\right) \backslash B,
$$

where $u \in\left(M_{x}\right)_{a(x)}, v \in\left(S_{x}\right)_{b(x)}, w \in\left(T_{x}\right)_{c(x)}$. Then there is a solution in the neighbourhood $V\left(x_{0}\right)$ of $x$, that is, there exists $x^{*} \in K \mid V\left(x_{0}\right)$ such that

$$
\left\langle N(u, v, w), \theta\left(y_{0}, g\left(x^{*}\right)\right)\right\rangle+\eta\left(g\left(x^{*}\right), y_{0}\right) \notin-\operatorname{int} C(x), \forall y_{0} \in K,
$$

where $u \in\left(M_{x}\right)_{a(x)}, v \in\left(S_{x}\right)_{b(x)}, w \in\left(T_{x}\right)_{c(x)}$.

Proof. Since $E$ is a Hausdorff topological vector space, $\theta$ and $g$ are locally non-positive about $g$ at $x_{0} \in K$.Without loss of generality,we can suppose that $V\left(x_{0}\right)$ is closed and convex, by Theorem 3.1, we know that the GVVLIFE has a solution in $K \mid V\left(x_{0}\right)$, that is, there exists $x^{*} \in K \mid V\left(x_{0}\right)$ such that

$$
\left\langle N(u, v, w), \theta\left(y, g\left(x^{*}\right)\right)\right\rangle+\eta\left(g\left(x^{*}\right), y\right) \notin-\operatorname{int} C\left(x^{*}\right), \forall y \in K \mid V\left(x_{0}\right) .
$$

Next, we prove that exists $x^{*} \in K \mid V\left(x_{0}\right)$ such that

$$
\left\langle N(u, v, w), \theta\left(y, g\left(x^{*}\right)\right)\right\rangle+\eta\left(g\left(x^{*}\right), y\right) \notin-\operatorname{int} C\left(x^{*}\right), \forall y \in K .
$$

If $x^{*} \in K \mathrm{I}$ int $V\left(x_{0}\right)$, then $V\left(x_{0}\right)-x^{*}$ is a neighbourhood of the origin and so it is absorbing. For any $y \in K$, there exists $\alpha \in(0,1)$ such that $\alpha\left(y-x^{*}\right) \in V\left(x_{0}\right)-x^{*}$, which means that $y_{\alpha}=\alpha y+(1-\alpha) x^{*} \in V\left(x_{0}\right) \mid K$. From (3), we get that

$$
\left\langle N(u, v, w), \theta\left(y_{\alpha}, g\left(x^{*}\right)\right)\right\rangle+\eta\left(g\left(x^{*}\right), y_{\alpha}\right) \notin-\operatorname{int} C\left(x^{*}\right) .
$$

Since $\theta$ is affine in the first argument, $\eta$ is convex in the second argument,we have

$$
\begin{aligned}
& \left\langle N(u, v, w), \theta\left(y_{\alpha}, g\left(x^{*}\right)\right)\right\rangle+\eta\left(g\left(x^{*}\right), y_{\alpha}\right) \\
& \in \alpha\left[\left\langle N(u, v, w), \theta\left(y, g\left(x^{*}\right)\right)\right\rangle+\eta\left(g\left(x^{*}\right), y\right)\right]+ \\
& (1-\alpha)\left[\left\langle N(u, v, w), \theta\left(x^{*}, g\left(x^{*}\right)\right)\right\rangle+\eta\left(g\left(x^{*}\right), x^{*}\right)\right]-C\left(x^{*}\right) .
\end{aligned}
$$

By Condition (i), we get

$$
\begin{aligned}
& \left\langle N(u, v, w), \theta\left(y_{\alpha}, g\left(x^{*}\right)\right)\right\rangle+\eta\left(g\left(x^{*}\right), y_{\alpha}\right) \\
& \in \alpha\left[\left\langle N(u, v, w), \theta\left(y, g\left(x^{*}\right)\right)\right\rangle+\eta\left(g\left(x^{*}\right), y\right)\right]-C\left(x^{*}\right) .
\end{aligned}
$$

Noting that

$$
\left\langle N(u, v, w), \theta\left(y, g\left(x^{*}\right)\right)\right\rangle+\eta\left(g\left(x^{*}\right), y\right) \notin-\operatorname{int} C\left(x^{*}\right) .
$$

In fact, if not, by (5), we get

$$
\begin{aligned}
& \left\langle N(u, v, w), \theta\left(y_{\alpha}, g\left(x^{*}\right)\right)\right\rangle+\eta\left(g\left(x^{*}\right), y_{\alpha}\right) \\
& \in \alpha\left[\left\langle N(u, v, w), \theta\left(y, g\left(x^{*}\right)\right)\right\rangle+\eta\left(g\left(x^{*}\right), y\right)\right]-C\left(x^{*}\right) \\
& \in-\operatorname{int} C\left(x^{*}\right)-C\left(x^{*}\right)=-\operatorname{int} C\left(x^{*}\right),
\end{aligned}
$$


which contradicts with (4), so (6) holds.

If $x^{*} \in K \mid b d V\left(x_{0}\right),(N, \theta, \eta)$ is said to be locally non-positive respect to $(M, S, T, g)$, then there exists $z_{0} \in K I$ int $V\left(x_{0}\right)$ such that

$$
\left\langle N(u, v, w), \theta\left(z_{0}, g\left(x^{*}\right)\right)\right\rangle+\eta\left(g\left(x^{*}\right), z_{0}\right) \in-C\left(x^{*}\right) .
$$

Similarly, for any $y \in K$, there exists $\beta \in(0,1)$ such that $\beta\left(y-z_{0}\right) \in V\left(x_{0}\right)-z_{0}$. Then $z_{\beta}=\beta y+(1-\beta) z_{0} \in V\left(x_{0}\right) \mid K$. It follows from (3) that

$$
\left\langle N(u, v, w), \theta\left(z_{\beta}, g\left(x^{*}\right)\right)\right\rangle+\eta\left(g\left(x^{*}\right), z_{\beta}\right) \notin-\operatorname{int} C\left(x^{*}\right) .
$$

Since $\theta$ is affine in the first argument, $\eta$ is convex in the second argument, we have

$$
\begin{aligned}
& \left\langle N(u, v, w), \theta\left(z_{\beta}, g\left(x^{*}\right)\right)\right\rangle+\eta\left(g\left(x^{*}\right), z_{\beta}\right) \\
& \in \beta\left[\left\langle N(u, v, w), \theta\left(y, g\left(x^{*}\right)\right)\right\rangle+\eta\left(g\left(x^{*}\right), y\right]+\right. \\
& (1-\beta)\left[\left\langle N(u, v, w), \theta\left(z_{0}, g\left(x^{*}\right)\right)\right\rangle+\eta\left(g\left(x^{*}\right), z_{0}\right)\right]-C\left(x^{*}\right) \\
& \in \beta\left[\left\langle N(u, v, w), \theta\left(y, g\left(x^{*}\right)\right)\right\rangle+\eta\left(g\left(x^{*}\right), y\right]-C\left(x^{*}\right) .\right.
\end{aligned}
$$

In addition, noting that

$$
\left\langle N(u, v, w), \theta\left(y, g\left(x^{*}\right)\right)\right\rangle+\eta\left(g\left(x^{*}\right), y\right) \notin-\operatorname{int} C\left(x^{*}\right) .
$$

In fact, if not, then by (9), we get

$$
\begin{aligned}
& \left\langle N(u, v, w), \theta\left(z_{\beta}, g\left(x^{*}\right)\right)\right\rangle+\eta\left(g\left(x^{*}\right), z_{\beta}\right) \\
& \subseteq \beta\left[\left\langle N(u, v, w), \theta\left(y, g\left(x^{*}\right)\right)\right\rangle+\eta\left(g\left(x^{*}\right), y\right)\right]-C\left(x^{*}\right) \\
& \subseteq-\operatorname{int} C\left(x^{*}\right)-C\left(x^{*}\right)=-\operatorname{int} C\left(x^{*}\right),
\end{aligned}
$$

which contradicts with (5), so (10) holds, the proof is completed.

\section{References}

[1]S.S. Chang, Salahuddin, M.K. Ahmad, X.R. Wang, Generalized vector variational like inequalities in fuzzy environment, Fuzzy Sets Syst. 2014.

[2]S.S. Chang, Y.G. Zhu, On variational inequalities for fuzzy mapping, Fuzzy Sets Syst. 32(1989) 359.

[3]H.H. Schaefer, Topological Vector Spaces, Graduate Texts In Mathematics. vol.Springer, New York. 1980.

[4]X.P. Ding, E. Tarafdar, Generalized variational-like inequalities with pseudo-monotone set valued mappings, Arch.Math. 74(2000)302-313.

[5] F. Ferro, A minimax theorem for vector-valued functions, J. Optim. Theory Appl. 60(1989)19-31.

[6]K. Fan, A generalization of Tychonoff' s fixed point theorem, Math. Ann. 142(1961)305-310.

[7]M.K. Ahmad, Salahuddin, R.U. Verma, Existence theorem for fuzzy mixed vector F-variational inequalities, Adv. Nonlinear Var.Inequal. 16(1)(2013)53-59.

[8]X.P. Ding, M.K. Ahmad, Salahudddin, Fuzzy generalized vector variational inequalities and complementarity problem, Nonlinear Funct.Anal. Appl. 13(2)(2008)253-263.

[9]M.F. Khan, S. Husain, Salahuddin, A fuzzy extension of generalized multivalued $\eta$-mixed vector variational-like inequalities on locally convex Hausdoff topological vector spaces, Bull. Calemts Math. Soc. 100(1)(2008)27-36.

[10]A. Kaufman, Introduction to the Theory of Fuzzy Subsets, Academic Press, New York. 1975.

[11]L.A. Zadeh, Fuzzy sets, inf. Control 8(1965)338-353. 